\title{
A Rare Case of Exophytic Duodenal Stromal Tumor: Case Report
}

\author{
Dr Manish K Singh, Dr Ramanuj Singh, Dr Prof Subrata Nag
}

\begin{abstract}
Gastrointestinal stromal tumors(GIST) have become a well established entity and its taxonomy is no more ambiguous but GIST of duodenum is rarely seen.In this paper, a patient presented with only recurrent pain abdomen and USG/CECT of abdomen revealed gallstone with $8 \times 7 \times 4.7 \mathrm{~cm}$ size mass lesion in the right hypochondrium, most likely arising from the second part of duodenum. The patient underwent cholecystectomy and wedge resection of tumor. The histopathology of the mass confirmed the diagnosis of duodenal stromal tumor.
\end{abstract}

\section{Introduction:}

Gastrointestinal stromal tumor (GIST) is the most common mesenchymal tumor of the gastrointestinal tract (GI) (1). GISTs arise most commonly in the stomach (60-80\%), followed by the small intestine (40\%) and then the colon, rectum or esophagus (2). The age range is generally 40 to 80 years at the diagnosis. Duodenum being the least common site. Duodenal GIST usually present with vague abdominal pain $(50 \%-70 \%)$ or they bleed into the lumen $(20 \%-50 \%)[3,4]$.. Small bowel GIST have a high propensity to exhibit malignant behavior[5]. Second part of the duodenum seems to be the common site of duodenal GIST and most of them will require pancreatcoduodenectomy for complete resection[6]. In patients with local disease, the recurrence rate is 35\% (1). A major diagnostic criterion of GISTs is expression of kit (CD117) and additional criteria include CD 34 and desmin $(7,8)$. In this case report, we reviewed the diagnosis, pathology and treatment of a patient of extra mural duodenal GIST.

\section{Case Report:}

A 32 years old female presented to us with history of recurrent episode of pain abdomen for last two months. She had no history of vomiting or malena . Clinical examination revealed a mild pale patient with tenderness in right hypochondrium. Except for low hemoglobin, her other hemogram and biochemistry were within normal levels. Ultrasound of abdomen reported multiple calculii in the gallbladder and exophytic mass between the gallbladder and the right kidney. CT demonstrated that there was a $8 \times 7 \times 4.7 \mathrm{~cm}$ size heterogeneously enhancing relatively defined mass lesion in the right hypochondrium most likely arising from the second part of duodenum and exophytically extending downward between the gallbladder, right kidney and liver (Figure 1). There was no definite infiltration into the adjacent organs or metastasis. Under this circumstances the patient underwent for laparotomy. On exploration we encounter multiple stones in the gallbladder along with exophytic mass of size approximately $9 \times 8 \times 5 \mathrm{~cm}$ arising from second part of duodenum. There was no local or distant metastsis or lymphadenopathy.The patient underwent cholecystectomy and wedge resection of tumor. In cut section the tumor was grey-whitish colored, rubbery with necrotic and cystic degeneration in same areas(Figure 2). The histopathology of the tumor reveals intersecting fasicles of spindle to oval cells(Figure 3 ). Mitosis is 5/50 and tumor cells are moderately pleomorphic. On immunohistochemistry, tumor cells strongly express C- Kit and S 100, focally express CD 34 and negative for SMA and H- caldesmon.

\section{Discussion:}

GISTs are a heterogenous group of diseases that differ in frequency, clinical symptoms and pathologic behavior in patients. Until recently it was categorized as a tumor of smooth muscle derivation. In the mid 1980s GIST has been suggested as a distinct entity. When localized GIST lesions tend to be primarily intramural and submucosal, often evading clinical diagnosis unless they rupture and bleed. Duodenal stromal tumor presents different clinical symptoms. One of most the important of them is acute hemorrhage into the intestinal tract due to necrosis of mucosa. It may be seen in up to $25 \%$ of GIST patient. GI bleeding can occur from ulcerating benign or malignant tumors. Several radiological techniques are used to image primary GISTs and metastatic lesions. These include double-contrast GI X-ray series with barium, endoscopic ultrasonography, computed tomography and magnetic resonance imaging (MRI) (9). Recently positron emission tomography scanning has been shown to be more sensitive than MRI. When GISTs are initially diagnosed, approximately $25 \%$ are frankly malignant. The criteria for this designation include metastasis or an invasion to adjacent organs. It is often difficult to predict the clinical behavior of GIST. Many pathologists believe that none of GISTs are truly benign. 
So terminology of the GISTs are being replaced "low risk" versus "high risk". Prognostic factors for high risk are large tumor size (>5 cm), many mitotic figures (> 5 per $50 \mathrm{HPF}$ ), fast growing and often infiltrating as in our case. The accurate diagnosis of GIST requires IHC staining for c-kit (CD 117) expression. Other antigenic markers (CD34, SMA, Desmin 2, S 100, Ki 67) are also variably positive. Surgical resection has been the mainstay of therapy for GIST $(10,11)$. Negative pathologic margins of resection generally is not difficult because GIST tends to hang from, not diffuse or infiltrate the organ of origin. Tumor rupture before or during resection is predictor of poor outcome. Local peritoneal tumor seeding is common. In a study, many patients (40\% in twenty four months) develop recurrent GIST despite complete resection of their primary tumor (4). The liver is the most common site of recurrence. Treatment options for recurrent and metastatic diseases have been limited. Clinical trials have shown that patients benefit from Imatinib therapy, so Imatinib was used in our case (12).

\section{Conclusion:}

This case of extra mural duodenal GIST is rare and effective surgical treatment allow better clinical outcome.

\section{References:}

[1]. Roberts PJ, Eisenberg B. Clinical presentation of gastrointestinal stromal tumors and treatment of operable disease. Eur J Cancer 2002;38 suppl 5:37-8.

[2]. Ignjatovic M. Gastrointestinal stromal tumors. Vojnosanit Pregl 2002;59:183-200.

[3]. Sturgeon C, Chejfec G, Espat NJ. Gastrointestinal stromal tumors: a spectrum of disease.Surg Oncol. 2003;12:21-26.

[4]. DeMatteo RP, Lewis JJ, Leung D, Mudan SS, Woodruff JM, Brennan MF. Two hundred gastrointestinal stromal tumors: recurrence patterns and prognostic factors for survival. Ann Surg. 2000;231:51-58.

[5]. DeMatteo RP, Heinrich MC, El-Rifai WM, Demetri G. Clinical management of gastrointestinal stromal tumors: Before and after STI-571. Human Pathol. 2002;33:466-67.

[6]. Winfield, Robert D, Hochwald, Steven N, Vogel, Stephen B, Hemming, Alan W, Liu, Chen , Cance , William G, Grobmyer , Stephen R. American Surgeon. 2006;72:719-23.

[7]. Rudolph P, Chiaravalli AM, Pauser U, et al. Gastrointestinal mesenchymal tumors-immunophenotypic classification and analysis. Wirchows Arch 2002;441:238-48.

[8]. Bergman J, O’Leary TJ. Gastrointestinal stromal tumors workshop. Hum Pathol 2001;23:578-82

[9]. Mehmet Yildirim, Saras Yakan, et al. A rare cause of intestinal hemorrhage: Stromal tumor of duodenum.Turk J Cancer 2004; 34(4): 163-165.

[10]. Pidhorecly I, Cheney RT, Kraybill WG, et al. Gastrointestinal stromal tumors: Current diagnosis biologic behavior and management. Ann Surg Oncol 2000;7:705-12.

[11]. Mosca F, Stracqualursi A, Persi A, et al. Our experience in the surgical treatment of gastrointestinal stromal tumors. Chir Ital 2001;53:809-20.

[12]. Maher MM, Yeo CJ, Lillemoe KD. Pancreas-sparing duodenectomy for infra-ampullary duodenal pathology Am J Surg 1996;171:62-7.

[13]. Demetri GD, Von Mehren M, Blanke CD, et al. Efficacy and safety of Imatinab mesylate in advanced Gastrointestinal stromal tumors. N Engl J Med 2002;347:472-80

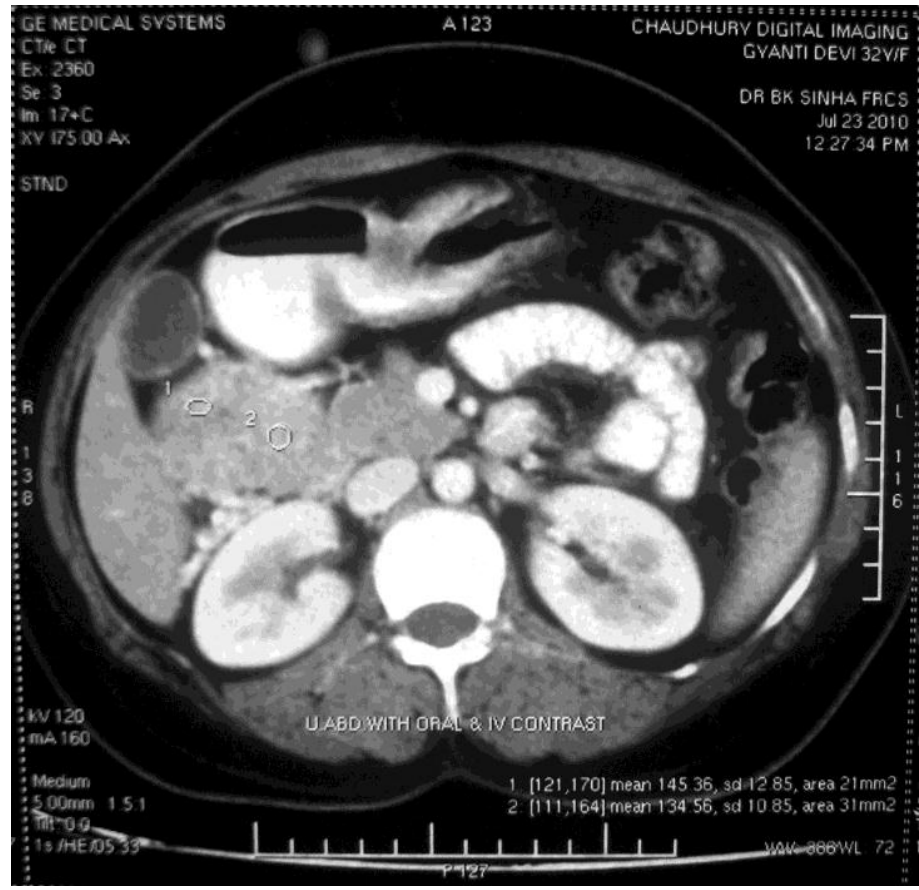




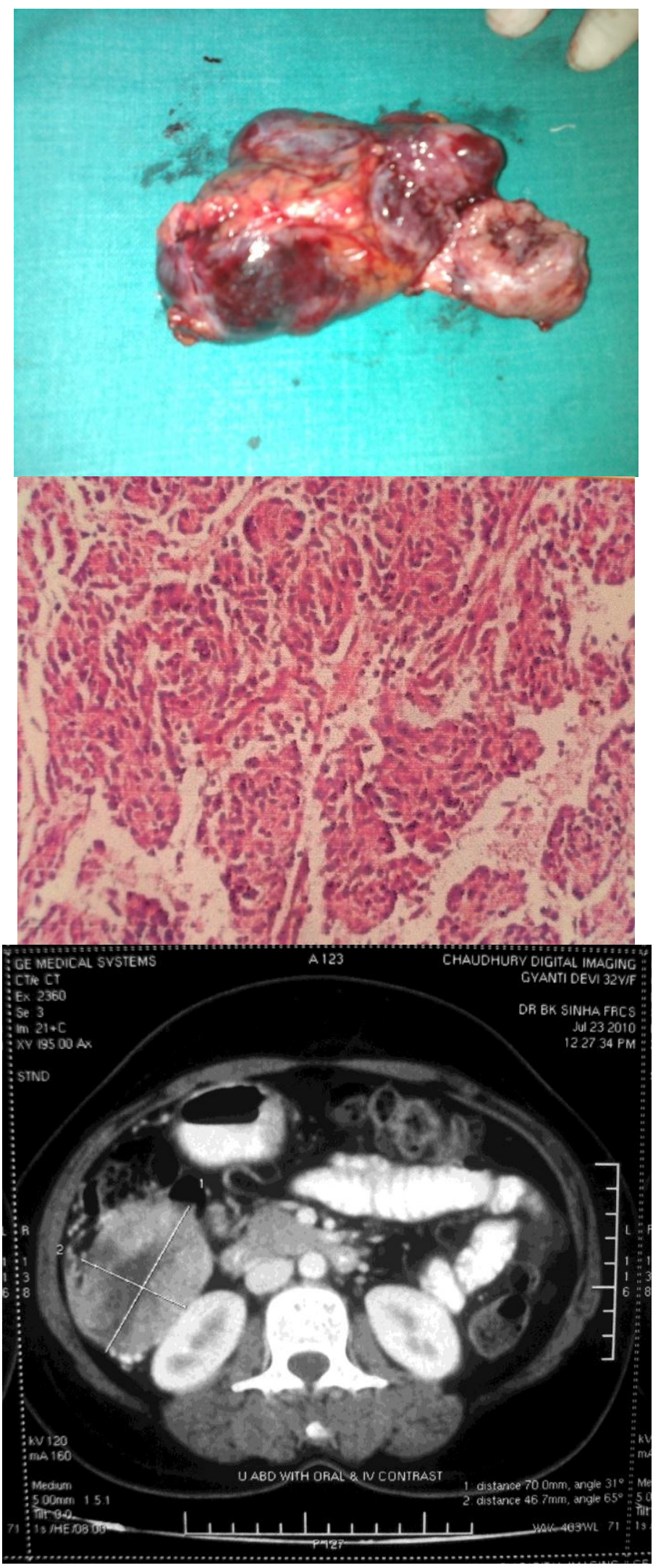

